\title{
PISTACIA TEREBINTHUS COFFEE PROTECTS AGAINST THIOACETAMIDE-INDUCED LIVER INJURY IN RATS
}

\author{
Ibrahim Halil Bahcecioglu', Murat Ispiroglu', Mehmet Tuzcu', Cemal Orhan ${ }^{3}$, Mustafa Ulas $^{4}$,

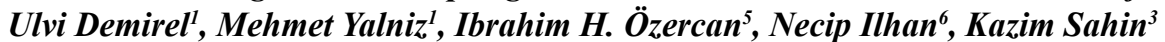

Firat University Faculty of Medicine, Elazig, Turkey: Department of Gastroenterology ${ }^{1}$, Department of Biology ${ }^{2}$, Department of Animal Nutrition ${ }^{3}$, Department of Physiology ${ }^{4}$, Department of Pathology ${ }^{5}$, Department of Biochemistry ${ }^{6}$

Summary: Aim/background: Pistacia terebinthus is used as a coffee substitute in the East and Southern Anatolia regions of Turkey. It contains unsaturated fatty acids, tocopherols, polyphenols and carotenoids. $P$. terebinthus has anti-inflammatory and potential antioxidant activity. In this study we evaluated the protective effects of $P$. terebinthus coffee (PTC) on thioacetamide (TAA)-induced liver injury in rats. Materials and methods: Twenty-eight male Sprague-Dawley rats were equally randomized into four groups. Chronic liver injury was induced with TAA $(100 \mathrm{mg} / \mathrm{kg}$ i.p. three times weekly). The first group of rats served as control and received only tap water $(\mathrm{G} 1)$, and the remaining groups of rats received PTC, p.o (G2); TAA (G3); TAA plus PTC, p.o (G4), respectively. Results: After 8 weeks, PTC intake significantly reduced fibrosis/ inflammation scores $(\mathrm{p}<0.05)$ in the livers of TAA-treated group. Compared to control group, PTC intake reduced transforming growth factor beta $($ TGF- $\beta)$ concentrations in the liver $(\mathrm{p}<0.05)$. Compared to the TAA group, TGF- $\beta$, nuclear factor kappa B (NF)- $\mathrm{B}(\mathrm{p}<0.05)$, tumor necrosis factor alpha (TNF- $\alpha)$ concentrations in the liver tissue were reduced by PTC intake. Discussion and conclusion: PTC intake provided beneficial effects against TAA-induced liver injury in rats. $P T C$ probably suppresses the proinflammatory cytokines through NF- $\kappa$ B signaling pathway.

Keywords: Liver fibrosis; Liver injury; Pistacia terebinthus; Inflammation

\section{Introduction}

Liver fibrosis is characterized by the accumulation of extracellular matrix proteins, such as collagen, due to alcohol intake, viruses and toxic chemicals. Hepatic stellate cells (HSC) play a key role in the development of liver fibrosis $(1,2)$. Thioacetamide (TAA) is a hepatotoxic agent that is frequently used in experimental studies and is able to cause centrilobular necrosis with a single dose. TAA-induced liver injury mainly occurs by oxidative stress mechanism (3). Pistacia terebinthus $L$. is a typical plant of the Mediterranean and West Asia regions. It is a member of Anacardiaceae family and is widely grown in the west and south regions of Turkey and known as terebinth. In various places all over the world, different organs of turpentine tree are used for multiple purposes. Archaeological findings show that turpentine has been used as a food since the ancient ages in Turkey. Offshoots and fruits are used in nutrition. Fruits are consumed as appetizers, in special village breads, and as coffee or tea (4). Pistacia terebinthus contains triterpenes and has been reported to have membrane stabilizing, antioxidant and anti-inflammatory effects (5-7).

In this study, we aimed to investigate the protective role of Pistacia terebinthus, which is derived from turpentine tree and widely consumed as coffee, against liver fibrosis.

\section{Material and methods}

This study used 28 Sprague-Dawley male rats with a mean weight of $250 \mathrm{~g}$. The study was performed at Experimental Research Center of Firat University, in accordance with the standard ethical rules of experimental animal research after the approval of Firat University Animal Experiments Ethics Committee. In line with ethical rules, the rats were kept at a temperature of $22 \pm 1{ }^{\circ} \mathrm{C}$ and on 12-hour light/dark cycle. The rats were fed on standard pellet chow and tap water. Weights of the rats were monitored over the course of the study.

The rats were randomized into 4 groups each containing 7 rats.

Group I (Control; $\mathbf{n}=7$ ): Basal diet plus intraperitoneal (i.p.) physiological saline for 8 weeks.

Group II (Pistacia terebinthus coffee; $\mathbf{n}=7$ ): Basal diet plus fresh-prepared terebinth coffee in drinking water plus i.p. physiological saline for 8 weeks.

Group III (Thioacetamide; $\mathbf{n}=7$ ): Basal diet plus i.p. $100 \mathrm{mg} / \mathrm{kg}$ TAA three times a week for 8 weeks.

Group IV (Thioacetamide plus Pistacia terebinthus coffee; $\mathbf{n}=7$ ): Basal diet plus i.p. $100 \mathrm{mg} / \mathrm{kg}$ TAA three times a week plus fresh-prepared terebinth coffee in drinking water for 8 weeks. 
The harvested wild-growing $P$. terebinthus fruits were cleaned in an air screen cleaner to remove immature and broken fruits and were spread out in the shade to dry for almost five days and then stored in polypropylene bags at room temperature. The dried fruits were roasted in a frying pan at $180^{\circ} \mathrm{C}$ with continuous stirring until the materials color becomes dark brown. Thereafter, the roasted plant pulverized with a garlic press to give a pasty consistency (8). Pistacia terebinthus, known as Harput Cedene Coffee in Elazig (East Anatolia area of Turkey) is sold like other coffee as roasted and squashed form in the market.

Since there is not a standard method for preparing Pistacia terebinthus coffee (PTC), the Turkish method of coffee preparation used in experimental studies was followed (9). The coffee was freshly prepared every two days. $5 \mathrm{~g}$ of $P T C$ and $10 \mathrm{~g}$ of sucrose were mixed in $60 \mathrm{ml}$ tap water, boiled, and waited until cooled. After separating the precipitated part at the bottom, its concentration in tap water was adjusted and then added into the drinking water of the rats.

In accordance with the procedure performed by Huber et al. (9), the content of coffee in drinking water was increased by $25 \%$ during each fresh coffee preparation. At the end of 7 days, the concentration reached up to $100 \%$. The coffee branded "Harput Çedene Coffee", which is sold in the markets, was used for coffee preparation. Thioacetamide (TAA, Sigma-Aldrich Chemical Co. USA) stock solution was prepared by dissolving $30 \mathrm{mg}$ pure TAA which is in crystal form in $100 \mathrm{~mL}$ distilled water $(0.03 \% \mathrm{w} / \mathrm{v})$ until all the crystals were dissolved. The solution was given to the rats intraperitoneally with TAA $100 \mathrm{mg} / \mathrm{kg}$ body-weight three times a week for 8 weeks. After the completion of 8 weeks, the rats were decapitated after 24 hours last dose under anesthesia following one-night fasting and blood samples were collected. Blood samples were centrifuged at $5000 \mathrm{rpm}$ for 5 minutes, and then stored at $-20^{\circ} \mathrm{C}$ until analysis. After the abdomens of the rats were opened, livers were removed while preserving tissue integrity and then they were weighed and recorded. Tissue samples were taken from different parts of the liver and fixed with $10 \%$ formalin solution and then paraffin blocks were prepared.

Serum alanine aminotransferase (ALT), aspartate aminotransferase (AST), gamma-glutamyl transferase (GGT), alkaline phosphatase (ALP) and total bilirubin levels were analyzed by Olympus AU 600 Auto-analyzer device using Olympus kits (Olympus Corp., Tokyo, Japan). Plasma malondialdehyde (MDA) levels were analyzed by Thiobarbituric acid method, which was modified by Yagi et al. (10) and Satoh et al. (11). Tumor necrosis factor alpha (TNF- $\alpha$ ) (Anti-TNF- $\alpha$ antibody, Abcam, Cambridge, UK), transforming growth factor beta (TGF- $\beta$ ) (Anti-TGF- $\beta$ antibody,

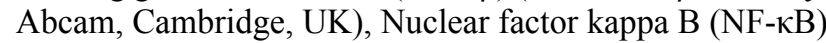
(Anti-NF- $\mathrm{B}$ p65 antibody, Abcam, Cambridge, UK), heme oxygenase-1 (HO-1) (Abcam, Cambridge, UK) and interleukin-6 (IL-6) (Santa Cruz Biotechnology, Inc. Santa Cruz, CA, USA) were studied by Western Blot method using kits.
Histopathological examination was done after $5 \mu \mathrm{m}$ liver sections were obtained from the paraffin-embedded blocks and stained with Hematoxylin\&Eosin (HE) for conventional histopathological examination. Masson Trichrome staining was used for the assessment of fibrosis. The stained preparations were blindly examined by an expert pathologist under a light microscope (Olympus BX-50, Tokyo, Japan) at $\times 40, \times 100, \times 200$ and $\times 400$ magnifications. Inflammatory cells were counted in at least 10 areas at high magnification (HPFX200 magnified) (12). The percentage of steatotic cells was assessed. For the assessment of necrosis, inflammatory foci in each $\mathrm{mm}^{2}$ were counted within at least 10 areas at high magnification (HPFX200 magnified) (11), and interpreted as balloon degeneration present/ absent. Tissue fibrosis was assessed according to the metavir scoring system (The French METAVIR Cooperative Study Group, 1994).

Score 0: No fibrosis

Score 1: Enlargement in portal regions without septa formation

Score 2: Enlargement in portal regions, sparse septa formation

Score 3: Remarkable septa formation, no cirrhosis

Score 4: Cirrhosis

\section{Statistical analysis}

Data of the groups were presented as mean \pm standard deviation. Statistical analysis was done using Statistical Package for the Social Sciences (SPSS) for Windows (version 13.0; SPSS Inc., Chicago, IL, USA). Kruskal-Wallis variance analysis and Mann-Whitney $U$ test were used for the evaluation of the data obtained from the parameters. A $p$ value $<0.05$ was considered significant.

\section{Results}

At baseline, the weight of the rats in all groups was similar $(p>0.05)$. At the end of study, the weights of the rats in the groups that were treated with TAA were significantly lower (Groups 3 and 4) as compared to that in the other two groups $(p \leq 0.05)$. Over the course of the study, 3 rats in the TAA group died (Group 3). No significant difference was observed between the groups regarding the serum AST, ALT and albumin levels $(\mathrm{p}>0.05)$ (Table 1).

Administration of TAA had caused a significant increase in TNF- $\alpha(\mathrm{p}<0.01$; Figure 1$)$, TGF- $\beta(\mathrm{p}<0.001$; Figure 2$)$, NF- $\kappa B(p<0.001$; Figure 3$)$, IL-6 ( $<<0.001$; Figure 5) levels with a decrease in HO-1 $(\mathrm{p}<0.001$; Figure 6$)$ compared to the normal- and PTC-treated groups. PTC significantly reduced the levels of TNF- $\alpha(\mathrm{p}<0.05)$, TGF- $\beta(\mathrm{p}<0.05)$, NF- $\kappa \mathrm{B}(\mathrm{p}<0.001)$, and IL-6 ( $\mathrm{p}<0.001)$ (Figure 1-3, 5), in addition to significant increase in HO-1 level $(\mathrm{p}<0.001)$ compared with the TAA group. TAA injection had caused a significant increase in MDA level $(p<0.01)$. However, no significant difference was found between TAA and TAA plus PTC groups in MDA levels ( $\mathrm{p}>0.05)$ (Figure 4). 
Tab. 1: Comparison of liver enzymes and albumin levels between the groups.

\begin{tabular}{|l|c|c|c|c|c|}
\hline & Control & PTC & TAA & TAA+PTC & p Value \\
\hline AST (IU/L) & $278 \pm 82$ & $259 \pm 103$ & $352 \pm 138$ & $288 \pm 99$ & 0.561 \\
\hline ALT (IU/L) & $106 \pm 36$ & $74 \pm 19$ & $106 \pm 37$ & $79 \pm 20$ & 0.141 \\
\hline Albumin (g/dl) & $3.1 \pm 0.38$ & $2.8 \pm 0.39$ & $3.7 \pm 0.85$ & $3.0 \pm 0.47$ & 0.840 \\
\hline
\end{tabular}

AST: Aspartate aminotransferase, ALT: Alanine aminotransferase, PTC: Pistacia terebinthus coffee, TAA: Thioacetamide

Tab. 2: Comparison of histopathological findings between the groups.

\begin{tabular}{|l|c|c|c|c|}
\hline & Control & PTC & TAA & TAA plus PTC \\
\hline Inflammation & $1.28 \pm 0.18$ & $1.83 \pm 0.30$ & $70 \pm 5.58$ & $38.85 \pm 2.00$ \\
\hline Necrosis & $0 \pm 0$ & $0 \pm 0$ & $10 \pm 1.35$ & $5.00 \pm 0.48$ \\
\hline Fibrosis & $0 \pm 0$ & $0 \pm 0$ & $2.5 \pm 0.28$ & $0.85 \pm 0.26$ \\
\hline Steatosis & $0 \pm 0$ & $0 \pm 0$ & $0 \pm 0$ & $0 \pm 0$ \\
\hline
\end{tabular}

PTC: Pistacia terebinthus coffee, TAA: Thioacetamide. Comparing TAA plus PTC with TAA, there was significant decrease in terms of inflammation $(\mathrm{p}<0.01)$, necrosis $(\mathrm{p}<0.001)$ and fibrosis $(\mathrm{p}<0.05)$

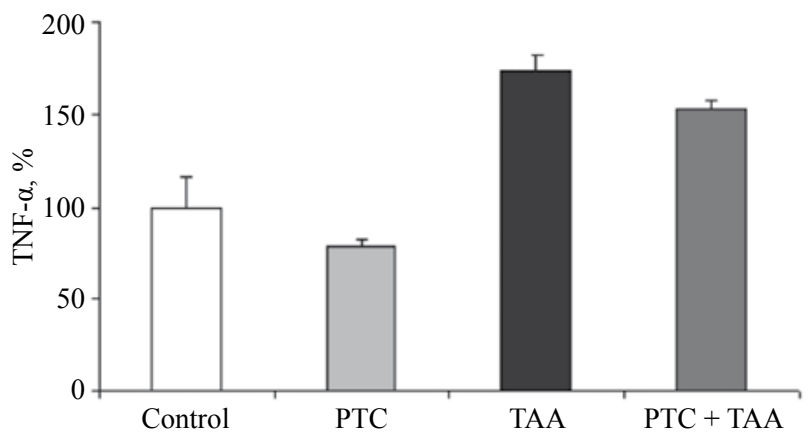

Fig. 1: Comparison of tumor necrosis factor alpha levels between the groups.

TNF- $\alpha$ : Tumor necrosis factor alpha, PTC: Pistacia terebinthus coffee, TAA: Thioacetamide

Control vs. Thioacetamide, $\mathrm{p}<0.01$; Control vs. TAA plus PTC, $\mathrm{p}<0.001 ;$ PTC vs. TAA, $\mathrm{p}<0.001 ;$ PTC vs. TAA plus PTC, $\mathrm{p}<0.001$; TAA vs. TAA plus PTC, $\mathrm{p}<0.05$.

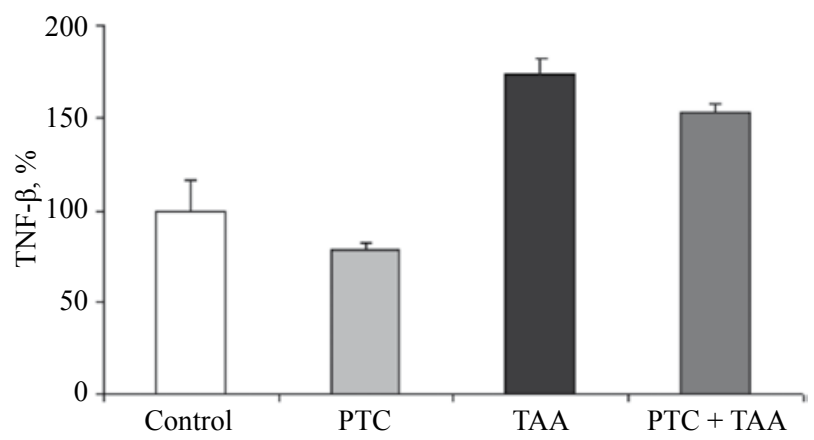

Fig. 2: Comparison of transforming growth factor beta levels between the groups.

TGF- $\beta$ : Transforming growth factor beta, PTC: Pistacia terebinthus coffee, TAA: Thioacetamide. Control vs. PTC, $\mathrm{p}<0.001$; Control vs. TAA, $\mathrm{p}<0.001$; Control vs. TAA plus PTC, $\mathrm{p}<0.001$; PTC vs. TAA, $\mathrm{p}<0.001$; PTC vs. TAA plus PTC, $\mathrm{p}<0.001$; TAA vs. TAA plus PTC, $\mathrm{p}<0.05$

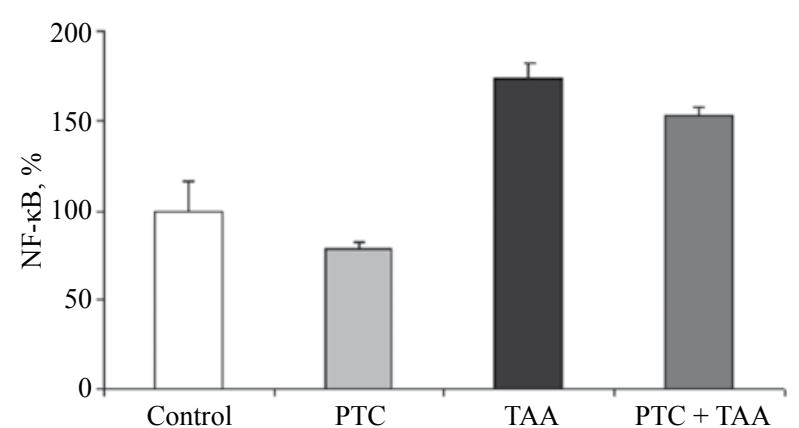

Fig. 3: Comparison of nuclear factor kappa B levels between the groups.

NF-אB: Nuclear factor kappa B, PTC: Pistacia terebinthus coffee, TAA: Thioacetamide

Control vs. PTC, $p>0.05$; Control vs. TAA, $p<0.001$; Control vs. TAA plus PTC, $\mathrm{p}<0.05$; PTC vs. TAA, $\mathrm{p}<0.001$; PTC vs. TAA plus PTC $\mathrm{p}>0.05$; TAA vs. TAA plus PTC, $\mathrm{p}<0.001$

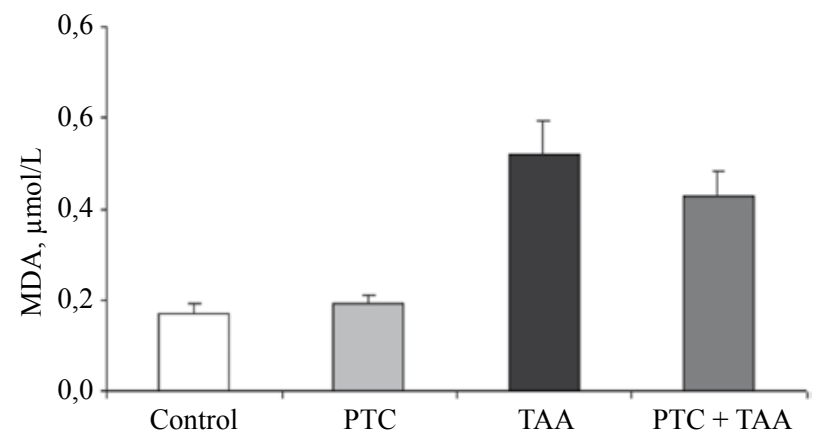

Fig. 4: Comparison of malondialdehyde concentration between the groups.

MDA: Malondialdehyde, PTC: Pistacia terebinthus coffee, TAA: Thioacetamide

Control vs. PTC, $\mathrm{p}>0.05$; Control vs. TAA, $\mathrm{p}<0.01$; Control vs. TAA plus PTC, $\mathrm{p}<0.05$; PTC vs. TAA, $\mathrm{p}<0.01 ;$ PTC vs. TAA plus PTC, $\mathrm{p}<0.05$; TAA vs. TAA plus PTC, $\mathrm{p}>0.05$. 


\section{Histopathological findings}

A significant decrease was observed in the inflammation, necrosis and fibrosis scores of the rats in TAA plus PTC group as compared to those in the TAA group. Histopathological findings are shown in Table 2 and Figure 7.

\section{Discussion}

TAA is a necrogenic and carcinogenic compound that contains thiono-sulphur (13-15). TAA is a potent hepatotoxic agent frequently used to develop a cirrhosis model in experimental animals. Following acute administration TAA leads to centrilobular necrosis and hepatitis $(15,16)$ and to hepatic cirrhosis in chronic use $(17,18)$. TAA-induced hepatic cirrhosis shows histopathological similarity to hepatic

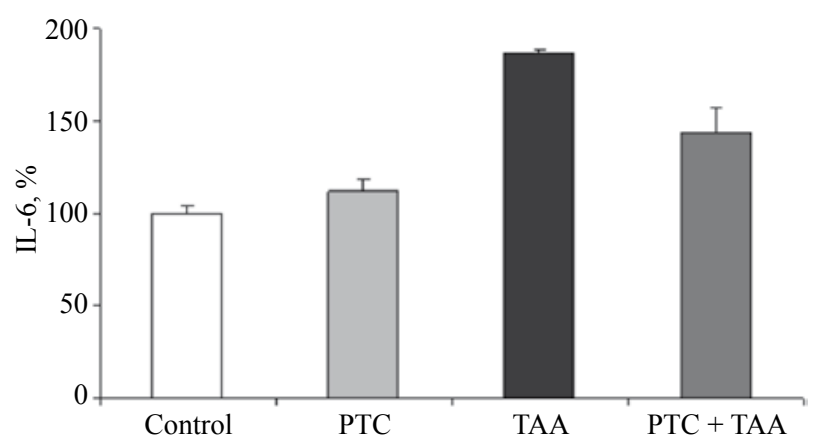

Fig. 5: Comparison of interleukin-6 levels between the groups. IL-6: Interleukin-6, PTC: Pistacia terebinthus coffee, TAA: Thioacetamide

Control vs. PTC, $\mathrm{p}<0.05$; Control vs. TAA, $\mathrm{p}<0.001$; Control vs. TAA plus PTC, $\mathrm{p}<0.001 ;$ PTC vs. TAA, $\mathrm{p}<0.001 ; P T C$ vs. TAA plus PTC, $\mathrm{p}<0.01$; TAA vs. TAA plus PTC, $\mathrm{p}<0.001$

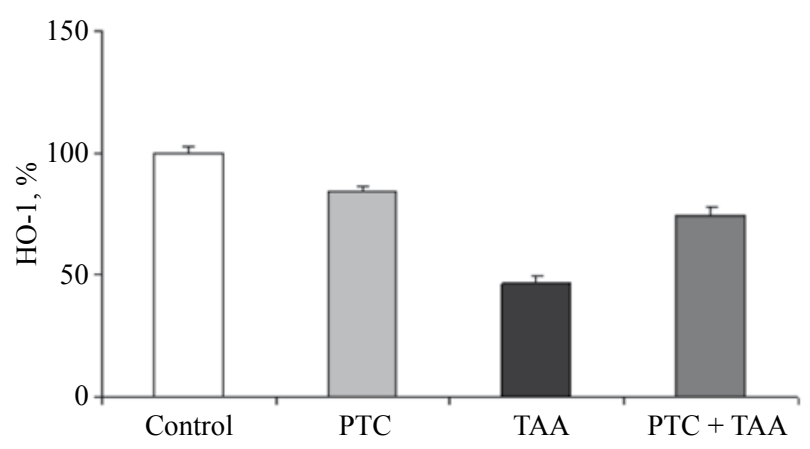

Fig. 6: Comparison of heme oxygenase-1 levels between the groups.

HO-1: Heme oxygenase-1, PTC: Pistacia terebinthus coffee, TAA: Thioacetamide

Control vs. PTC, $\mathrm{p}<0.001$; Control vs. TAA, $\mathrm{p}<0.001$; Control vs. TAA plus PTC, $\mathrm{p}<0.001$; PTC vs. TAA, $\mathrm{p}<0.001 ;$ PTC vs. TAA plus PTC, $\mathrm{p}<0.05$; TAA vs. TAA plus PTC, $\mathrm{p}<0.001$. cirrhosis that result from viral hepatitis (19). With increasing information on the pathophysiology of fibrosis, the opinion that cirrhosis might be a treatable disease has strengthened. In addition to numerous pharmacological studies on this subject, natural antioxidant compounds are among the treatment methods that have become an agenda in the recent years. In this study, we induced chronic liver injury by administering TAA for 8 weeks.

Pistacia terebinthus (Çedene, Terebinth) is a typical plant of Mediterranean and West Asia regions. In Turkey, it is grown in the coastal regions and on the hills or in pine woods on Taurus Mountains at $1600 \mathrm{~m}$ height (20). In the east and southeast of Turkey, it is eaten after being roasted, or roasted fruits are consumed as coffee after being exposed to various procedures. In Elazig region, it is traditionally prepared and drunk, and is known as 'Çedene coffee'. In the present study, we give the rats Pistacia terebinthus coffee to drink. Whilst there was no death in the group that received $P T C$, three rats in the TAA groups died. This might be associated with the protective effect of PTC. Although not significant, there was a decrease in AST and ALT levels in PTC groups Pistacia terebinthus is a member of the Anacardiaceae family which contains potentially toxic substances like tannin-containing leaves. Hence, toxicological adverse events might be encountered by administration of this subject. However, as mentioned above no death occurred in the group that received PTC and biochemical analysis did not reveal any abnormality indicating a toxic injury to the liver. Similarly, no toxic effect was shown in a previous study (21).

PTC provided a significant decrease in inflammation, necrosis and fibrosis scores. The present study is the first study that investigated the effects of PTC on hepatic fibrosis. TAA leads to hepatic injury by transformation to its harmful metabolites and by covalently binding to hepatic macromolecules as a result of intensive metabolic processes mediated by microsomal monooxygenase enzyme system, which requires nicotinamide adenine dinucleotide phosphate (NADPH) and cytochrome P450 (22-24). In the liver, TAA is first metabolized into TAA sulfoxide by CYP2E1. TAA sulfoxide (TASO) is then changed into TAA S-dioxide $\left(\mathrm{TASO}_{2}\right)$ by further metabolism. Mainly, these two compounds cause injury in the liver. TASO leads to cell death, decrease in mitochondrial activity, increase in nucleus volume and intracellular concentration of calcium ion, and alterations in cell membrane permeability (25). Chemical contents of Pistacia terebinthus include fatty acids, tocopherols and sterols. Oleic acid is the major fatty acid, accounting for $43-51 \%$ of the fatty acids. Active vitamin E content of Pistacia terebinthus was determined to be $396.8-517.7 \mathrm{mg} / \mathrm{kg}$ and $\alpha$ and $\gamma$ tocopherol, which are among predominant isomers, content was determined to be $110-150 \mathrm{mg} / \mathrm{kg}$. It is known that $\beta$ sitosterol is the major sterol, accounting for $80 \%$ (1341-1802 mg/kg) of the sterols (26).

A study found that Pistacia terebinthus extract has 12 times higher antioxidant capacity as compared to butyl- 

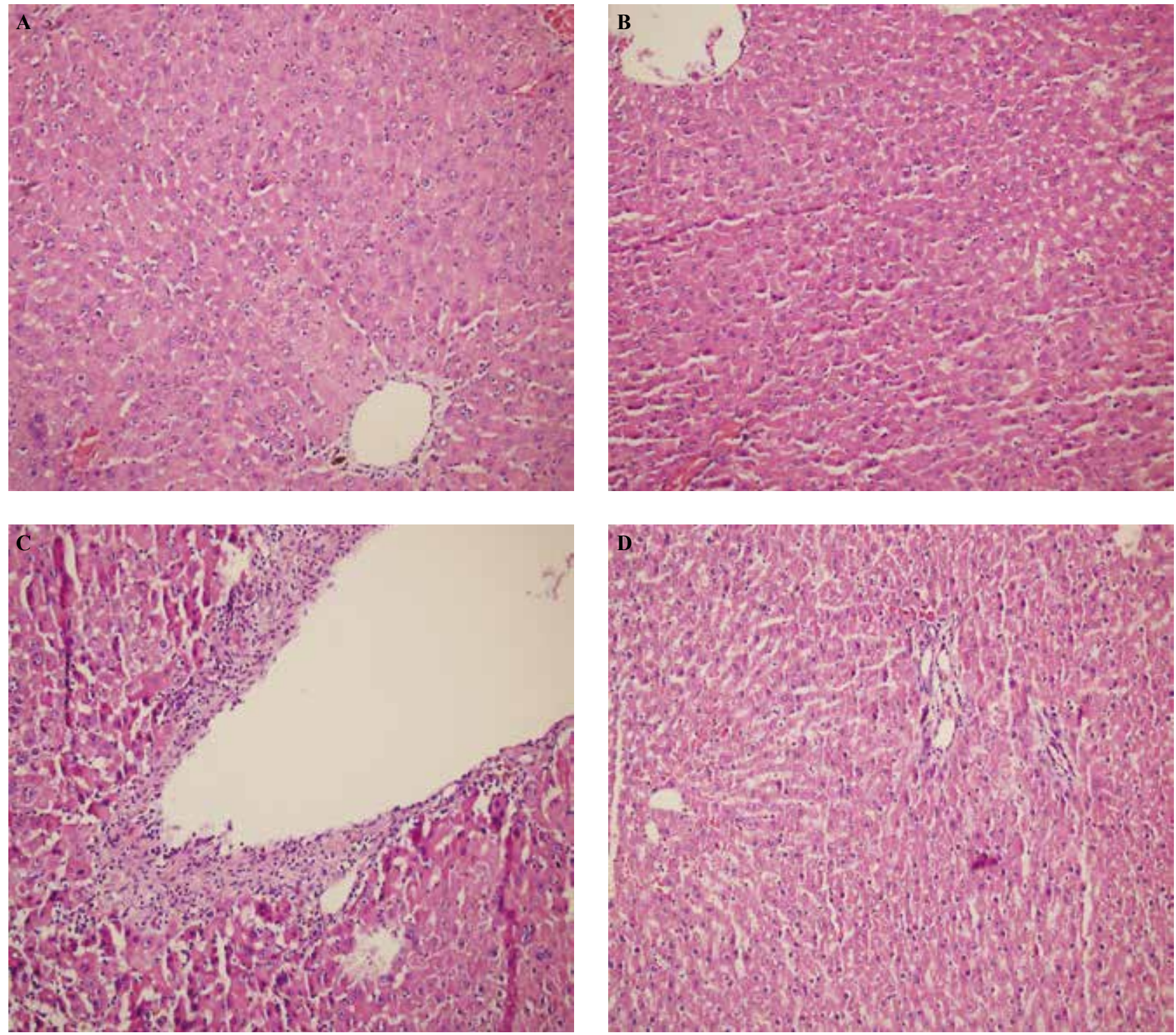

Fig. 7: Hematoxylin \& Eosin staining. (A) Control group (HEX200); (B) Pistacia terebinthus coffee (PTC) (HEX200); (C) Thioacetamide (TAA) treated rats (HEX200; significant increase in fibrous tissue and inflammatory activity); (D) TAA plus PTC rats (HEX200; Sparse inflammatory cell infiltration in portal area).

ated hydroxyanisole and ascorbic acid (20). This, probably, is because of phenol and high flavonoid components. Membrane stabilizing effect of alpha tocopherol is well known (27). PTC, by also stabilizing biological membranes with its rich alpha tocopherol content, shows hepatoprotective activity However, the amount of the ingredients of the raw plant undergo to changes during the preparation of the seeds. Roasting causes some desirable and/or undesirable changes in physical, chemical and nutritional properties of the seeds and extracted oils. One of the main desired outcomes of roasting process is the increase in antioxidant activity. It was shown that oils obtained from roasted seeds and nuts are more resistant to the oxidative deteriorations (28). Hence, it is hard to conclude that antioxidant and hepatoprotective effect of this plant mixture is simply caused only by one tocopherols also could be due to triterpenes, namely by oleanic acid; which is considered very important. It was observed that MDA formation is enhanced and glutathione level is decreased in the hepatic cells following TAA administration (29-31). Free radical-induced lipid peroxidation as well causes progression of TAA-induced hepatic fibrosis. In the present study, we determined increased HO-1 levels and decreased MDA levels in the rats receiving PTC. This suggests that $P T C$ reduces oxidative stress by its membrane stabiliz- 
ing effects and has protective efficacy. NF- $\kappa \mathrm{B}$ is a nuclear transcriptional activator and plays a central role in inflammation and in the response to stress (32). With PTC, which has been suggested to have anti-inflammatory efficacy in the previous studies (6), we determined a significant decrease in NF- $\kappa$ B, TNF- $\alpha$, TGF- $\beta$ and IL- 6 proinflammatory and fibrogenic cytokine levels. Anti-inflammatory effect of $P T C$ is probably associated with the inhibition of NF- $\kappa \mathrm{B}$ activity.

In conclusion, $P T C$ has a preventive effect against experimental hepatic fibrosis. This effect seems to be associated with potent antioxidant and anti-inflammatory efficacy. PTC particularly prevents the production of proinflammatory and fibrogenic cytokines probably by inhibiting NF- $\kappa$ B activity and thus, suppresses fibrosis formation.

\section{References}

1. Chen IS, Chen YC, Chou CH, Chuang RF, Sheen LY, Chiu CH. Hepatoprotection of silymarin against thioacetamide-induced chronic liver fibrosis. J Sci Food Agric 2011; 92: 1441-1447.

2. Abramovitch S, Dahan-Bachar L, Sharvit E et al. Vitamin D inhibits proliferation and profibrotic marker expression in hepatic stellate cells and decreases thioacetamide-induced liver fibrosis in rats. Gut 2011 Dec; 60(12): 1728-1737.

3. Stankova P, Kucera O, Lotkova H, Rousar T, Endlicher R, Cervinkova Z. The toxic effect of thioacetamide on rat liver in vitro. Toxicol In Vitro 2010; 24, 20972103 4. Walheim, L. 1981. Western Fruit and Nuts. HP Books, Inc., Tucson, Arizona, p. 166

5. Giner-Larza EM, Manez S, Giner RM et al. Anti-inflammatory triterpenes from Pistacia terebinthus galls. Planta Med 2002; 68: 311315

6. Giner-Larza EM, Manez S, Recio MC et al. Oleanonic acid, a 3-oxotriterpene from Pistacia, inhibits leukotriene synthesis and has anti-inflammatory activity. Eur J Pharmacol 2001; 428: 137143 .

7. Matthaus, B, Ozcan MM. Quantitation of fatty acids, sterols, and tocopherols in turpentine (Pistacia terebinthus Chia) growing wild in Turkey. J Agric Food Chem 2006; 54: 76677671 .

8. Gogus F, Ozel MZ, Kocak D, Hamilton JF, Lewis AC. Analysis of roasted and unroasted Pistacia terebinthus volatiles using direct thermal desorption-GCxGCTOF/MS. Food Chemistry 2011; 129: 1258-1264.

9. Huber WW, Scharf G, Nagel G, Prustomersky S, Schulte-Hermann R, Kaina B Coffee and its chemopreventive components Kahweol and Cafestol increase the activity of O6- methylguanine-DNA methyltransferase in rat liver-comparison with phase II xenobiotic metabolism. Mutat Res 2003; 522: 57.

10. Yagi K, Matsuoka S, Linnane AW, Zimmet P. Plasma lipid peroxide levels in an urbanized Micronesian population-Nauru. J Nutr Sci Vitaminol (Tokyo) 1981; 27 425428.

11. Satoh K, Takamatsu S, Sakuta S, Mizuno S, Metoki H, Takamatsu M. Augmented malondialdehyde production by platelets from patients with cerebrovascular disorders. Jpn Circ J 1981; 45: 13351341.

12. Koca SS, Bahcecioglu IH, Poyrazoglu OK, Ozercan IH, Sahin K, Ustundag B. The treatment with antibody of TNF-alpha reduces the inflammation, necrosis and fibrosis in the non-alcoholic steatohepatitis induced by methionine- and choline deficient diet. Inflammation 2007; 31: 9198.
13. Landon EJ, Naukam RJ, Rama Sastry BV. Effects of calcium channel blocking agents on calcium and centrilobular necrosis in the liver of rats treated with hepatotoxic agents. Biochem Pharmacol 1986; 35: 697-705.

14. Kizer DE, Clouse JA, Ringer et al. Assessment of rat liver microsomal epoxide hydrolase as a marker of hepatocarcinogenesis. Biochem Pharmacol 1985; 34: $1795-1800$.

15. Müller A, Machnik F, Zimmermann T, Schubert $H$. Thioacetamide-induced cirrhosis-like liver lesions in rats usefulness and reliability of this animal model. Exp Pathol 1988; 34: 229-236.

16. Ali SO, Darwish HA, Ismail NA. Modulatory effects of curcumin, silybin-phytosome and alpha-R-lipoic acid against thioacetamide-induced liver cirrhosis in rats. Chem Biol Interact 2014; 5: 26-33.

17. Abdulaziz Bardi D, Halabi MF, Abdullah NA, Rouhollahi E, Hajrezaie M, Abdulla MA. In vivo evaluation of ethanolic extract of Zingiber officinale rhizomes for its protective effect against liver cirrhosis. Biomed Res Int 2013; 2013: 918460

18. Salama SM, Abdulla MA, Alrashdi AS, Hadi AH. Mechanism of hepatoprotective effect of boesenbergia rotunda in thioacetamide-induced liver damage in rats. Evid Based Complement Alternat Med 2013; 2013: 157456.

19. Moustafa AH, Ali EM, Moselhey SS, Tousson E, El-Said KS. Effect of coriander on thioacetamide-induced hepatotoxicity in rats. Toxicol Ind Health 2014; 30: 621 .

20. Kavak, DD, Altiok E, Bayraktar O, Ulkü S. Pistacia terebinthus extract: As a potential antioxidant, antimicrobial and possible-glucuronidase inhibitor. J Mol Cata. B: Enzym 2010; 64: 167-171.

21. Bakirel T, Sener S, Bakirel U, Keles, Sennazli G, Gurel A. The Investigation of the Effects of Pistacia terebinthus L. Upon Experimentally Induced Hypercholesterolemia and Atherosclerosis in Rabbits. Turk. J. Vet. Anim Sci 2003; 27: $1283-1292$.

22. Hunter AL, Holscher MA, Neal RA. Thioacetamide-induced hepatic necrosis I. Involvement of the mixed-function oxidase enzyme system. J Pharmacol Exp Ther 1977; 200: 439-448.

23. Chieli E, Malvaldi G. Role of the microsomal FAD-containing monooxygenase in the liver toxicity of thioacetamide-S-oxide. Toxicology 1984; 31: 41-52.

24. Porter WR, Neal RA. Metabolism of thioacetamide and thioacetamide S-oxide by rat liver microsomes. Drug Metab Dispos 1978; 6: 379388.

25. Bruck R, Aeed H, Avni Y et al. Melatonin inhibits nuclear factor kappa B activation and oxidative stress and protects against thioacetamide induced liver damage in rats. J Hepatol 2004; 40: 8693.

26. Matthaus B, Ozcan MM. Quantitation of fatty acids, sterols, and tocopherols in turpentine (Pistacia terebinthus Chia) growing wild in Turkey. J Agric Food Chem 2006; 54: 76677671.

27. Niki E, Traber MG. A history of vitamin E Ann. Nutr. Metab. 2012; 61: 207212

28. Durmaz G, Gokmen V. Changes in oxidative stability, antioxidant capacity and phytochemical composition of Pistacia terebinthus oil with roasting. Food Chemistry $2011 ; 128: 410-414$.

29. Diez-Fernandez C, Bosca L, Fernandez-Simon L, Alvarez A, Cascales M. Relationship between genomic DNA-ploidy and parameters of liver damage during necrosis and regeneration induced by thioacetamide. Hepatology 1993; 18: 912-918.

30. So EC, Wong KL, Huang TC, Tasi SC, Liu CF. Tetramethylpyrazine protects mice against thioacetamide-induced acute hepatotoxicity. J Biomed Sci 2002; 9 : $410-414$.

31. Sanz N, Diez-Fernandez C, Andres D, Cascales M. Hepatotoxicity and aging endogenous antioxidant systems in hepatocytes from 2-, 6-, 12-, 18- and 30-monthold rats following a necrogenic dose of thioacetamide. Biochim Biophys Acta 2002; 1587, 12-20.

32. Calzado MA, Bacher S, Schmitz ML. NF-kappaB inhibitors for the treatment of inflammatory diseases and cancer. Curr Med Chem 2007; 14: 367-376.

Received: $27 / 07 / 2014$

Accepted in revised form: 28/05/2015

\section{Corresponding author:}

Ibrahim Halil Bahcecioglu, Gastroenteroloji Kliniği, Firat Universitesi Tip Fakultesi, Elazig, Turkey; e-mail: ihbahcecioglu (a)yahoo.com 